\title{
Quantum harmonic oscillator with time dependent mass
}

\author{
I. Ramos-Prieto, ${ }^{1}$ A. Espinosa-Zuñiga, ${ }^{1}$ M. Fernández-Guasti, ${ }^{2}$ \\ and H.M. Moya-Cessa ${ }^{1}$ \\ ${ }^{1}$ INAOE, Coordinacin de Optica, Apdo. Postal 51 y 216, 72000 Puebla, Pue., Mexico. \\ ${ }^{2}$ Depto. de Fsica, CBI, Universidad Autnoma Metropolitana - Iztapalapa, 09340, \\ CDMX, Ap. Postal 55-534, Mexico.
}

\begin{abstract}
We use the Fourier operator to transform a time dependent mass quantum harmonic oscillator into a frequency dependent one. Then we use Lewis-Ermakov invariants to solve the Schrödinger equation by using squeeze operators. Finally we give two examples of time dependencies: quadratically and hyperbolically growing masses.
\end{abstract}

PACS numbers: 42.50.Ct, 42.50.-p, 42.50.Pq, 42.50.Dv 


\section{Introduction}

The harmonic oscillator equation with time dependent parameters [1-8] has been solved for a sudden frequency change using a continuous treatment based on an invariant formalism [9]. This analytic treatment requires that the time dependent parameter is a monotonic function whose variation is short compared with the typical period of the system. This procedure allowed us to obtain analytic solutions that do or do not exhibit squeezing [10-14] depending on time when departing from an initial coherent state.

Ion laser interactions [15] is one of the fields where time dependent harmonic oscillator arise and some solutions have been obtained 16, 17 for the time dependent ion-laser Hamiltonian [18].

On the other hand, time dependent mass harmonic oscillators have been considered, probably the most famous of them being the so-called Caldirola-Kanai oscillator [19, 20] that has been studied extensively over the years [21-24]. A time dependent exponentially growing mass is considered in this oscillator, while the stiffness is also allowed to change in time. We have considered recently a time dependent mass subject to a sudden change [25] while keeping the stiffness parameter constant.

Propagation in one dimensional optical metamaterials with graded refractive index can be efficiently treated with the time dependent harmonic oscillator formalism. The time axis is replaced by the wave direction of propagation and the medium inhomogeneity is represented by the time dependent parameter. Optical metamaterials are relevant for photonic crystals and other state of the art optical applications [26, 27].

Mass reconfiguration is being used to manipulate oscillations [28, 29] and chaotic behaviour in macroscopic oscillators [30].

\section{Time dependent mass}

The harmonic Hamiltonian with time dependent mass $M(t)$ reads

$$
\hat{H}(t)=\frac{1}{2}\left[\frac{\hat{p}^{2}}{M(t)}+\kappa \hat{q}^{2}\right]
$$

where $\kappa$ is the stiffness. We want to solve the Schrödinger equation

$$
i \frac{\partial|\psi(t)\rangle}{\partial t}=\hat{H}(t)|\psi(t)\rangle
$$

where we have set $\hbar=1$. We consider the Fourier operator 3134

$$
\hat{\mathcal{F}}=\exp \left(-i \frac{\pi}{4}\left[\hat{p}^{2}+\hat{q}^{2}\right]\right) e^{i \pi / 4}
$$

and do the transformation $|\psi(t)\rangle=\hat{\mathcal{F}}|\Psi(t)\rangle$. Inserting this expression in (4)

$$
i \frac{\partial|\Psi(t)\rangle}{\partial t}=\frac{1}{2} \hat{\mathcal{F}}^{\dagger}\left[\frac{\hat{p}^{2}}{M(t)}+\kappa \hat{q}^{2}\right] \hat{\mathcal{F}}|\Psi(t)\rangle
$$

by rescaling time with the stiffness constant, $\tau=\kappa t$ we rewrite the Schrödinger equation in the form

$$
i \frac{\partial|\Psi(\tau)\rangle}{\partial \tau}=\frac{1}{2} \hat{\mathcal{F}}^{\dagger}\left[\frac{\hat{p}^{2}}{\kappa M(\tau)}+\hat{q}^{2}\right] \hat{\mathcal{F}}|\Psi(\tau)\rangle
$$


By noting that $e^{-i \frac{\theta}{2}\left(\hat{p}^{2}+\hat{q}^{2}\right)} \hat{q} e^{i \frac{\theta}{2}\left(\hat{p}^{2}+\hat{q}^{2}\right)}=\hat{q} \cos \theta-\hat{p} \sin \theta$ and $e^{-i \frac{\theta}{2}\left(\hat{p}^{2}+\hat{q}^{2}\right)} \hat{p} e^{i \frac{\theta}{2}\left(\hat{p}^{2}+\hat{q}^{2}\right)}=$ $\hat{p} \cos \theta+\hat{q} \sin \theta$ we obtain

$$
i \frac{\partial|\Psi(\tau)\rangle}{\partial \tau}=\frac{1}{2}\left[\hat{p}^{2}+\frac{\hat{q}^{2}}{\kappa M(\tau)}\right]|\Psi(\tau)\rangle
$$

\section{Invariants and solutions}

It is well-known that the above equation has an invariant of the form [1]

$$
\hat{I}=\frac{1}{2}\left(\frac{\hat{q}^{2}}{\rho^{2}}+(\rho \hat{p}-\dot{\rho} \hat{q})^{2}\right)
$$

where the auxiliary function $\rho$, obeys the Ermakov equation

$$
\ddot{\rho}+\frac{\rho}{\kappa M(\tau)}=\frac{1}{\rho^{3}}
$$

and is related to another auxiliary function $u$ by the equations

$$
u=\rho \cos \left(\int \frac{d \tau}{\rho^{2}}\right), \quad \rho=u \sqrt{1+\left(\int \frac{d \tau}{u^{2}}\right)^{2}}
$$

where $u$ obeys the equation

$$
\ddot{u}+\frac{u}{\kappa M(\tau)}=0 .
$$

By using the unitary operator $\hat{T}$

$$
\hat{T}=e^{i \frac{\ln \rho}{2}\left(\hat{q} \hat{p}+\hat{p} \hat{q}+\frac{2 \rho \dot{\rho}}{\rho^{2}-1} \hat{q}^{2}\right)} .
$$

that may be rewritten as (see the Appendix)

$$
\hat{T}=e^{i \frac{\ln \rho}{2}(\hat{q} \hat{p}+\hat{p} \hat{q})} e^{-i \frac{\dot{\rho}}{2 \rho} \hat{q}^{2}},
$$

we can relate the invariant, $\hat{I}$, to the time independent harmonic oscillator Hamiltonian,

$$
\hat{T} \hat{I} \hat{T}^{\dagger}=\frac{1}{2}\left(\hat{p}^{2}+\hat{q}^{2}\right) .
$$

This relation hints us that the transformation operator 12 may be relevant in the solution of (6). Indeed, it has been shown that the Schrödinger equation for the time dependent harmonic Hamiltonian has a solution of the form 9

$$
|\Psi(t)\rangle=e^{-i \hat{I} \int_{0}^{\tau} \omega(t) d t} \hat{T}^{\dagger} \hat{T}(0)|\Psi(0)\rangle .
$$

with the characteristic frequency of the time dependent harmonic oscillator defined by 25$] \omega(t)=1 / \rho^{2}$. 

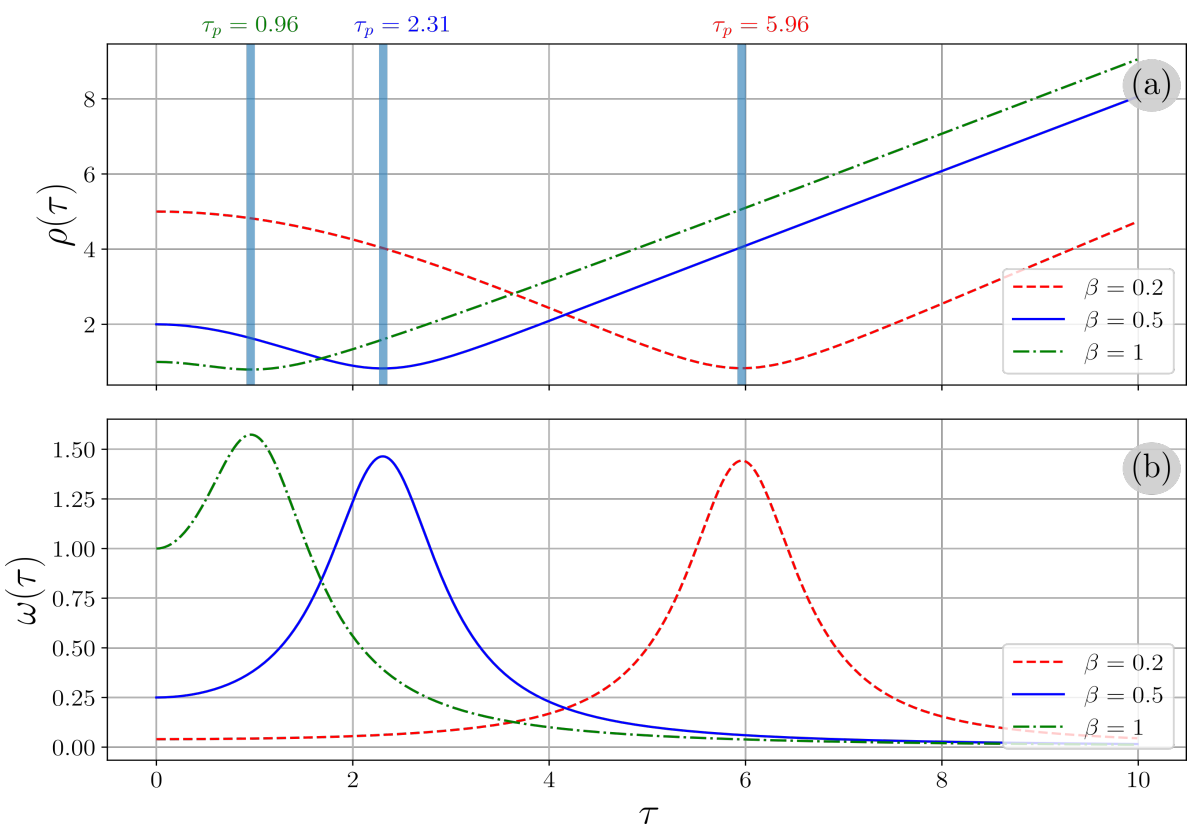

Figure 1. We plot the functions (a) $\rho(\tau)$ and (b) $\omega(\tau)$ for $\beta=0.2$ (dot-dashed line), $\beta=0.5$ (dashed line) and $\beta=1$ (solid line).

\subsection{Hyperbolically growing mass}

We consider now a time dependent mass of the form $M(\tau)=\frac{\cosh ^{2}(\beta \tau)}{2 \beta^{2} \kappa}$. For this choice we obtain the solution $u=\tanh (\beta \tau)$ and therefore the auxiliary function, $\rho$, is given by

$$
\rho=\tanh \beta \tau \sqrt{1+\frac{[\beta \tau-\operatorname{coth}(\beta \tau)]^{2}}{\beta^{2}}},
$$

and the integral in one of the exponentials of the wavefunction in equation (20) may be calculated by the relation

$$
\int \omega(\tau) d \tau=\cos ^{-1} \frac{1}{\sqrt{1+\frac{[\beta \tau-\operatorname{coth}(\beta \tau)]^{2}}{\beta^{2}}}}
$$

If we consider the initial state to be a coherent state

$$
|\psi(0)\rangle=|\alpha\rangle=e^{-\frac{|\alpha|^{2}}{2}} \sum_{n=0}^{\infty} \frac{\alpha^{n}}{\sqrt{n !}}|n\rangle=\hat{D}(\alpha)|0\rangle
$$

where $\hat{D}(\alpha)$ is the so-called Glauber displacement operator 35

$$
\hat{D}(\alpha)=e^{\alpha \hat{a}^{\dagger}-\alpha^{*} \hat{a}}
$$

with the annihilation and creation operators given by

$$
\hat{a}=\frac{1}{\sqrt{2}}(\hat{q}+i \hat{p}), \quad \hat{a}^{\dagger}=\frac{1}{\sqrt{2}}(\hat{q}-i \hat{p})
$$

and where the states $|n\rangle$ are Fock states. Then the Fourier transformed initial wavefunction becomes $|\Psi(0)\rangle=|-i \alpha\rangle$ and so the evolved state has the form

$$
|\Psi(\tau)\rangle=\hat{T}^{\dagger}\left|-i \alpha e^{-i \int_{0}^{\tau} \omega(t) d t}\right\rangle,
$$


or, more explicitely

$$
|\Psi(\tau)\rangle=e^{i \frac{\dot{\rho}}{2 \rho} \hat{q}^{2}} e^{-i \frac{\ln \rho}{2}(\hat{q} \hat{p}+\hat{p} \hat{q})}\left|-i \alpha e^{-i \int_{0}^{\tau} \omega(t) d t}\right\rangle,
$$

this is the application of squeeze operators to a coherent state, i.e, a squeezed state 10 14]. For this particular example, figure 1 (a) shows that there is an specific time, $\tau_{p}$, for which the derivative of the function $\rho(\tau)$ has a minimum, and therefore $\dot{\rho}\left(\tau_{p}\right)=0$, for which we obtain the exact squeezed state

$$
\left|\Psi\left(\tau_{p}\right)\right\rangle=\left|-i \alpha e^{-i \int_{0}^{\tau} \omega(t) d t}, \ln \rho\left(\tau_{p}\right)\right\rangle,
$$

and therefore, going back to the original picture, by transforming this state with the inverse Fourier operator we obtain finally

$$
\left|\psi\left(\tau_{p}\right)\right\rangle=\left|i \alpha e^{-i \int_{0}^{\tau} \omega(t) d t}, \ln \rho\left(\tau_{p}\right)\right\rangle .
$$

In figure 1 we also show a plot of the characteristic frequency of the time dependent harmonic oscillator.
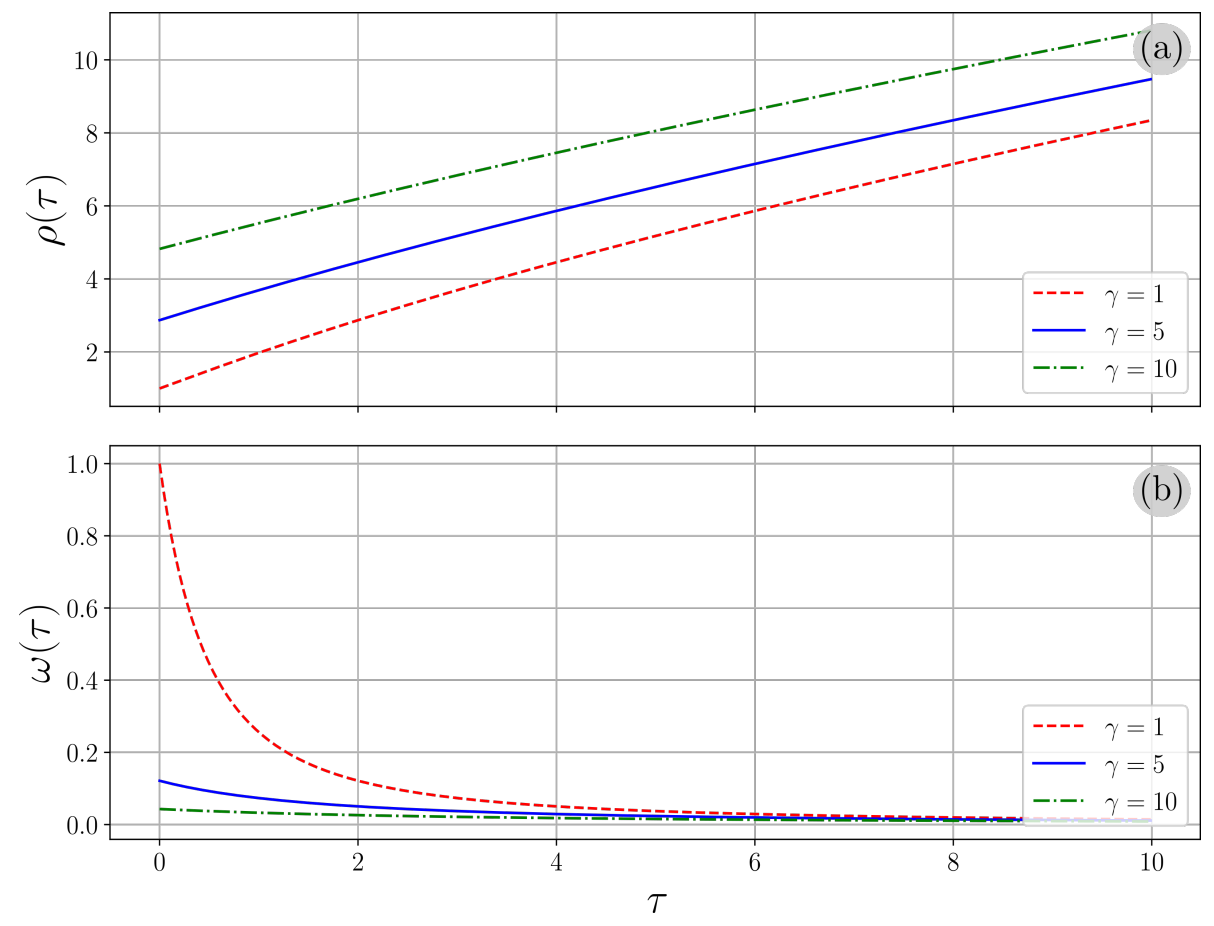

Figure 2. We plot the functions (a) $\rho(\tau)$ and (b) $\omega(\tau)$ for $\gamma=1$ (dot-dashed line), $\gamma=5$ (dashed line) and $\gamma=10$ (solid line).

\subsection{Quadratically growing mass}

We consider now a mass that evolves as

$$
\kappa M(\tau)=(\gamma+2 \tau)^{2}
$$

the solution to 10 reads then

$$
u(\tau)=\sqrt{\gamma+2 \tau}
$$


and therefore the auxiliary Ermakov function reads

$$
\rho(\tau)=\sqrt{(\gamma+2 \tau)\left[1+\frac{1}{4} \ln ^{2}(\gamma+2 \tau)\right]}
$$

in this case, the integral in on of the exponentials of equation $(20)$ is given by

$$
\int \omega(\tau) d \tau=\cos ^{-1} \frac{1}{\sqrt{1+\frac{1}{4} \ln ^{2}(\gamma+2 \tau)}}
$$

In figure 2 we show the behaviour of (a) the auxiliary function $\rho(\tau)$ and (b) the characteristic frequency of the time dependent harmonic oscillator. In this case there is no way to simplify the state to an ideal squeezed state as the function $\rho(\tau)$ does not show either maximums nor minimums.

\section{Conclusions}

It has been shown that if the Fourier operator is used to transform the quantum time dependent harmonic oscillator Hamiltonian when a time dependent mass is considered, the resultant Hamiltonian takes the form of one with time dependent frequency. By writing the invariant for this latter Hamiltonian, we can generate solutions that involve squeeze operators. We have given two examples of time dependent mass, namely hyperbolically and quadratically growing masses. Finally, by using the relation (9), we have been able to show how to calculate the integral of the characteristic time dependent frequency needed in (20).

\section{Appendix}

The exponential

$$
e^{i \frac{\ln \rho}{2}\left(\hat{q} \hat{p}+\hat{p} \hat{q}+\frac{2 \rho \dot{\rho}}{1-\rho^{2}} \hat{q}^{2}\right)}
$$

has the sum of the operators

$$
\hat{A}=i \frac{\ln \rho}{2}(\hat{q} \hat{p}+\hat{p} \hat{q})
$$

and

$$
\hat{B}=i \ln \rho \frac{\rho \dot{\rho}}{\rho^{2}-1} \hat{q}^{2}
$$

We can show that the commutators of the above operators is

$$
[\hat{A}, \hat{B}]=2 \ln \rho \hat{B} \text {. }
$$

Using the fact that the commutator of the two operators is proportional to one of them, we can factor the exponential in the form

$$
e^{i \frac{\ln \rho}{2}\left(\hat{q} \hat{p}+\hat{p} \hat{q}+\frac{2 \rho \dot{\rho}}{1-\rho^{2}} \hat{q}^{2}\right)}=e^{i \frac{\ln \rho}{2}(\hat{q} \hat{p}+\hat{p} \hat{q})} e^{-i \frac{\dot{\rho}}{2 \rho} \hat{q}^{2}}
$$


[1] Lewis H R 1967 Phys. Rev. Lett. 18 510-512

[2] Lewis H R and Riesenfeld W B 1969 J. Math. Phys. 101458

[3] Dodonov V V and Man'ko V I 1979 Phys. Rev. A 20 550-561

[4] Ray J R 1982 Phys. Rev. A 26729

[5] Janszky J and Yushin Y Y 1986 Opt. Commun. 59 151- 154

[6] Pedrosa I A 1997 Phys. Rev. A 553219

[7] Haas F and Goedert J 2001 Phys. Lett. A 279 181-188

[8] Bouquet S and Lewis H R 1996 J. Math. Phys. 37 5509-5517

[9] Fernández Guasti M and Moya-Cessa H 2003 Phys. Rev. A 67063803

[10] Yuen H P 1976 Phys. Rev. A 132226

[11] Caves C M 1981 Phys. Rev. D 231693

[12] Satyanarayana M V, Rice P, Vyas R and Carmichael H J 1989 J. Opt. Soc. Am. B 6228

[13] Moya-Cessa H and Vidiella-Barranco A 1992 J. of Mod. Opt. 39 2481-2499

[14] Loudon R and Knight P L 1987 J. of Mod. Opt. 34709

[15] Schleich W P "Quantum Optics in Phase Space" (Wiley-VCH, 2001)

[16] Schrade G, Man'ko V I, Schleich W P and Glauber R J 1995 Quantum Semiclass. Opt. 7307

[17] Schrade G, Bardroff P J, Glauber R J, Leichtle C, Yakovlev V and Schleich W P 1997 Appl. Phys. $B \mathbf{6 4} 181$

[18] Moya-Cessa H M, Soto-Eguibar F, Vargas-Martinez J M, Juarez-Amaro R and Zuñiga-Segundo A 2012 Phys. Rep. 513 229-261

[19] Caldirola P 1941 Il Nuovo Cimento 18 393-400

[20] Kanai E 1950 Prog. Theor. Phys. 3 440-442

[21] Daneshmand R and Tavassoly M K 2017 Int. J. of Theor. Phys 56 1218-1232

[22] Daneshmand R and Tavassoly M K 2016 Las. Phys. 26065204

[23] Kim S P 2003 J. of Phys. A 36 12089-12095

[24] Man'ko V I and Safonov S S J. of Russian Las. Res. 20 119-139

[25] Moya-Cessa H and Fernández Guasti M 2007 Rev. Mex. Fís. 53 42-46

[26] Shvartsburg A B and Maradudin A A "Waves in Gradient Metamaterials" (World Scientific, 2013.)

[27] Mazharimousavi S H, Roozbeh A and Halilsoy M 2013 J. of Electr. Waves and App. 27 2065-2074

[28] Zevin A A and Filonenko L A 2007 J. of App. Math. and Mech. 71 892-904

[29] Wright J A, Bartuccelli M and Gentile G 2017 J. of Math An. and App. 449 1684-1707

[30] Stilling D S D and Szyszkowski W 2002 Int. J. of Non-Linear Mech. 37 89-99

[31] Namias V 1980 J. Appl. Math. 25241

[32] Agarwal G S and Simon R 1994 Opt. Commun. 11023

[33] Fan H-Y and Chen J-H 2015 Front. Phys. 10100301

[34] Weimann S, Perez-Leija A, Lebugle M, Keil R, Tichy M, Gräfe M, Heilmann R, Nolte S, MoyaCessa H, Weihs G, Christodoulides D N and Szameit A 2016 Nature Comm. 711027

[35] Glauber R J 1963 Phys. Rev. 1312766 